Bull. Chem. Soc. Ethiop. 2019, 33(1), 103-112.

ISSN 1011-3924

(c) 2019 Chemical Society of Ethiopia and The Authors

Printed in Ethiopia

DOI: https://dx.doi.org/10.4314/bcse.v33i1.10

\title{
CHARACTERIZATION OF MOLYBDENUM AND TUNGSTEN PHOSPHATES COMPOUNDS PREPARED USING AMMONIUM PHOSPHATE SALT AS FLUX
}

\author{
Trevor T. Chiweshe* \\ Institute for Groundwater Studies, University of the Free State, Nelson Mandela Drive, \\ Bloemfontein, 9300, South Africa
}

(Received June 14, 2018; Revised January 25, 2019; Accepted January 30, 2019)

\begin{abstract}
Fusion of powdered molybdenum and tungsten oxide using ammonium phosphate salt as flux (ratio: 1:25 respectively) yielded soluble products which were unstable. The yellow (Mo) and purple-blue (W) solutions in deionized water, methanol and acetone decolorized within 3-5 hours. Infrared analysis of molybdenum and tungsten products revealed characteristic broad bands bearing two minor peaks in the region of $1040-1130 \mathrm{~cm}^{-1}$ which reflects the simultaneous existence of $\mathrm{PO}_{2}$ and $\mathrm{PO}_{3}$ groups. SEM-EDX quantitative analysis results showed percentage weight of the identified elements in the increasing order of Mo or W $<$ P O with molybdenum product having $7.1 \% \mathrm{wt}(\mathrm{Mo}), 37.2 \% \mathrm{wt}(\mathrm{P})$ and $53.5 \% \mathrm{wt}(\mathrm{O})$ whilst tungsten product had $10.3 \%$ wt $(\mathrm{W}), 24.7 \%$ wt $(\mathrm{P})$ and $46.5 \%$ wt $(\mathrm{O})$. The EDX results indicated the presence of polyphosphate products whilst the SEM images showed microscopic crystalline product of molybdenum and a hygroscopic product of tungsten. XRD patterns of both molybdenum and tungsten products corresponded to the standard (JCPDS) pattern of molybdenum oxide phosphate $\mathrm{MoO}_{2}\left(\mathrm{PO}_{3}\right)_{2}$ and tungsten oxide phosphate $\left(\mathrm{WO}_{2}\left(\mathrm{PO}_{3}\right)_{2}\right)$, respectively.
\end{abstract}

KEY WORDS: Molybdenum, Tungsten, Ammonium phosphate flux, Polyphosphates

\section{INTRODUCTION}

Metaphosphates belong to a series of polyphosphates (general formula $\mathrm{M}\left(\mathrm{PO}_{3}\right)_{3} \mathrm{M}=$ trivalent elements) which are characterized by a tridimensional network of isolated $\mathrm{MO}_{6}$ octahedral connected through a infinite number of repeated $\mathrm{PO}_{4}$ tetrahedral units. These compounds owing to their interesting properties have found numerous applications in anticorrosive pigments, heterogeneous catalysis, ionic exchange, optoelectronics, glass host laser and optical amplifier technologies due to their novel magnetic properties [1, 2]. The solid state chemistry of metaphosphates compounds is considered unusual due to the trivalent oxidation state of the transition metals [3]. Numerous polyphosphate compounds of transition, lanthanides and rare earth metals have been reported [4] and are composed of different polymeric structures that exhibits either antiferromagnetic or ferromagnetic properties [5].

Different techniques of preparing polyphosphates have been reported [6]. The solid-state [7] and microwave [8] methods are the preferred methods due to better yields $(>90 \%)$. However, these techniques are not without disadvantages, the synthesis of micro-crystalline metaphosphates using the solid-state technique often takes long up to 7 days whilst microwave technique requires at least 6 days of product formation [9]. Although these techniques have been shown to be effective, there still remains a need to optimize these techniques and shorten the preparation time.

A recent study by Chiweshe et al. [10] shows an alternative approach of isolating microcrystalline chromium metaphosphates from chromite mineral ores using ammonium phosphate salt as flux at moderate temperature $\left(800^{\circ} \mathrm{C}\right)$. The $\mathrm{XRD}$ analysis identified the isolated microcrystals as C-type chromium metaphosphate, $\mathrm{Cr}\left(\mathrm{PO}_{3}\right)_{3}$ (JCPDS: \#01-077-0672) [11, 12]. The isolation of $\mathrm{Cr}\left(\mathrm{PO}_{3}\right)_{3}$ from the chromite mineral ore using the fusion technique prompted further research in establishing an alternative method of synthesizing molybdenum and tungsten metaphosphate compounds using the same technique. Most of the well-investigated chromium

*Corresponding author. E-mail: chiweshetrevor@gmail.com

This work is licensed under the Creative Commons Attribution 4.0 International License 
and molybdenum metaphosphates belong to the $\mathrm{C}$-form type which is isostructural to the iron metaphosphate compound [13]. However, information on tungsten metaphosphates is very scarce with little or no evidence of its existence.

The aim of this research study was to synthesize metaphosphates compounds of molybdenum and tungsten using ammomonium phosphate salt as flux. The objective was to determine if micro-crystalline metaphosphate products of molybdenum and tungsten can be obtained using the fusion technique. Characterization using infrared (IR), scanning electron microscopy (SEM), energy dispersive X-ray spectroscopy (EDX) and X-ray powder diffraction (XRD) was conducted in order to determine the chemical composition of the obtained products.

\section{EXPERIMENTAL}

Chemicals, instrumentation and procedures

Chromium(VI) oxide $\left(\mathrm{CrO}_{3}\right)$, molybdenum powder (Mo), tungsten(VI) oxide $\left(\mathrm{WO}_{3}\right)$, ammonium dihydrogen phosphate $\left(\left(\mathrm{NH}_{4}\right) \mathrm{H}_{2} \mathrm{PO}_{4}\right)(99 \%)$ and anhydrous sodium sulfite $(98 \%)$ were purchased from Merck. Ceramic crucibles (capacity, $150 \mathrm{~mL}$ ) and the UV-vis quartz cuvettes were purchased from Terra Nova Ceramics (South Africa) and Lasec SA, respectively. Methanol, dichloromethane (DCM), chloroform, ethyl acetate, acetone together with the mineral acids, $\mathrm{HCl}(10 \mathrm{M})$ and $\mathrm{HNO}_{3}(15 \mathrm{M})$ were purchased from Sigma-Aldrich. The volumetric flasks used in this research study were of Blaubrand grade (A) type and the glass beakers were of the Schott Duran type. Ultra-pure deionised water (conductivity, $0.01 \mu \mathrm{S} / \mathrm{cm}$ ) was used for all experimental analyses. All the experimental results are as reported as an average of at least 3 replicates.

\section{Instrumentation}

Barnstead Thermolyne furnace (max. temperature $1300{ }^{\circ} \mathrm{C}$ ) was used for the sample fusion procedure whilst the Eutech CyberScan ( $\mathrm{pH}$ 1500) was used for $\mathrm{pH}$ measurement of the solutions. Characterization of the isolated chromium, molybdenum and tungsten products were done on a Digilab (FTS 2000) infrared (IR) spectrometer, an Oxford X-Max ${ }^{\mathrm{N}}$ energy dispersive $\mathrm{X}$-ray spectrometer (EDX) equipped with a Tescan VEGA3 scanning electron microscope (SEM) (Centre of Microscopy, University of the Free State) and Bruker-D8 Advance X-ray diffractometer $(\mathrm{XRD})$ with a $\mathrm{Cu}-\mathrm{K}$ radiation $(\approx 0.154 \mathrm{~nm})$ in the $20-60^{\circ}$ range (Physics Department, University of the Free State).

Preparation of chromium, molybdenum and tungsten-metaphosphates compounds using ammonium phosphate flux

Chromium metaphosphate was prepared from fusion of chromium(III) oxide $\left(\mathrm{CrO}_{3}\right)$ with ammonium phosphate flux as described by Chiweshe et al. [10]. Powdered samples of molybdenum (Mo powder) and tungsten(VI) oxide $\left(\mathrm{WO}_{3}\right)$ were mixed thoroughly in separate ceramic crucibles with ammonium phosphate in the ratio of 1:25 respectively. The samples were placed in a pre-heated furnace $\left(800^{\circ} \mathrm{C}\right)$ and heated until red-hot molten melt was obtained $(-25$ min). The resultant green (Cr), yellow (Mo) and purple-blue (W) glassy melts were cooled and dissolved in deionised water. The dissolution of chromium melt resulted in instant precipitation of chromium which was filtered and dried at room temperature. Molybdenum and tungsten melts yielded yellow and purple-blue solutions respectively that were different from the solid chromium product. Both molybdenum and tungsten solutions decolourised overnight after left to crystallize at room temperature. 
$U V$-vis determination of factors influencing decolourization of molybdenum and tungsten solutions using different solvents

Fusion of Mo powder and $\mathrm{WO}_{3}$ with ammonium phosphate flux was repeated as described in the preceding section. The resultant dark yellow and purple-blue melts of molybdenum and tungsten respectively were tested for solubility in polar and non-polar solvents. Molybdenum melt was soluble in deionised water and methanol whilst tungsten melt was soluble in deionised water, methanol and acetone. Chromium precipitate (chromium metaphosphate) was insoluble in both polar and non-polar solvents. Aliquots of the yellow and purple-blue solutions of Mo and $\mathrm{W}$ respectively were transferred into separate quartz cuvettes and determined for the maximum absorbance $\left(\lambda_{\max }\right)$. Factors influencing decolourization (Figures 1-3) were determined by examining the decrease in absorbance of both solutions in different solvents.

\section{$U V$-vis analysis of the isolated chromium metaphosphate solutions}

The isolated chromium metaphosphate product was fused with sodium phosphate flux using the ratio of 1:25 respectively. The resultant melt was cooled at room temperature and dissolved in deionised water $(20 \mathrm{~mL})$ to yield a clear green solution. Aliquots of the green solution were analysed using UV-vis spectra and the results are shown in Figure 4.

Comparison of the IR, SEM-EDX and XRD results of chromium metaphosphate, molybdenum and tungsten phosphate products

Isolation of solid products of molybdenum and tungsten. The dark yellow (Mo) and purple-blue (W) melts were dissolved in a minimum volume $(10 \mathrm{~mL})$ of dried methanol (dried using anhydrous sodium phosphate) to yield a super-saturated solution. Both solutions were dried rapidly by blowing nitrogen gas into the solutions. The resultant solid products were kept in a desiccator filled with nitrogen gas for 48 hours before characterized using IR (Figure 5). The IR spectra of molybdenum and tungsten were compared with the chromium metaphosphate spectrum to determine any similarities in the stretching frequencies. Further characterizations of molybdenum and tungsten products were done using SEM-EDX (Figures 6) and XRD (Figure 7).
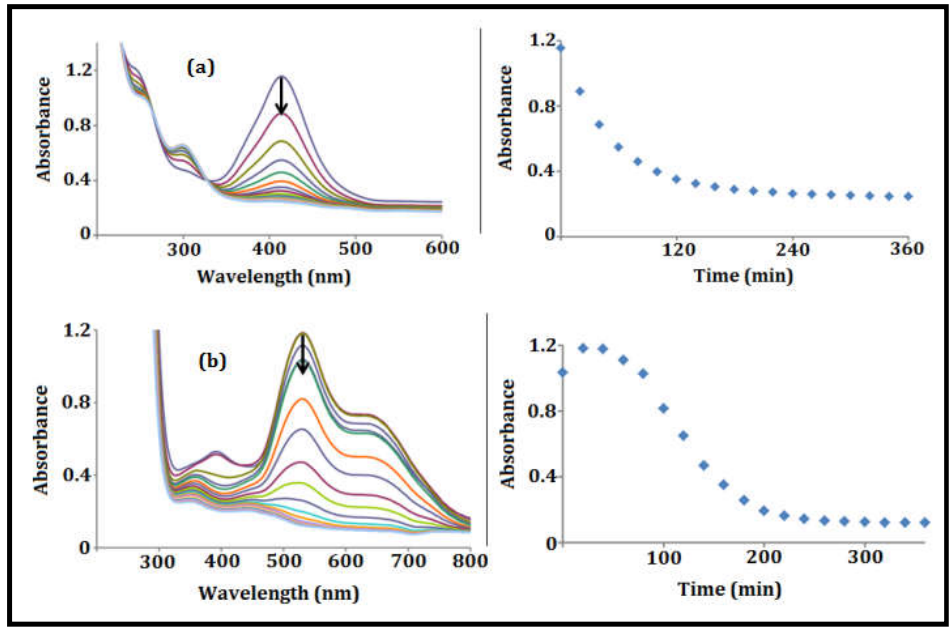

Figure 1. UV-vis analysis of decolourization of (a) the yellow solution (Mo) at 5 min time interval $\left(\lambda_{\max }=415 \mathrm{~nm}\right)$ and (b) the purple-blue solution $(\mathrm{W})$ in deionised water at 5 min time interval $\left(\lambda_{\max }=535 \mathrm{~nm}\right)$.

Bull. Chem. Soc. Ethiop. 2019, 33(1) 

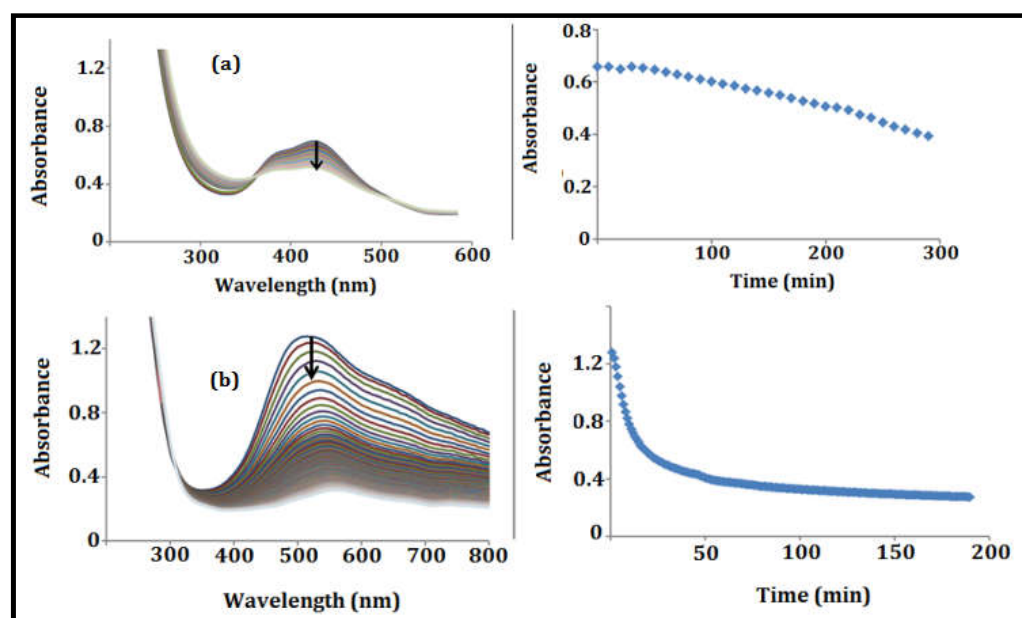

Figure 2. UV-vis analysis of decolourization of (a) the yellow solution (Mo) at 10 min time interval $\left(\lambda_{\max }=420 \mathrm{~nm}\right)$ and (b) the purple solution (W) in methanol at 2 min time interval $\left(\lambda_{\max }=515 \mathrm{~nm}\right)$.

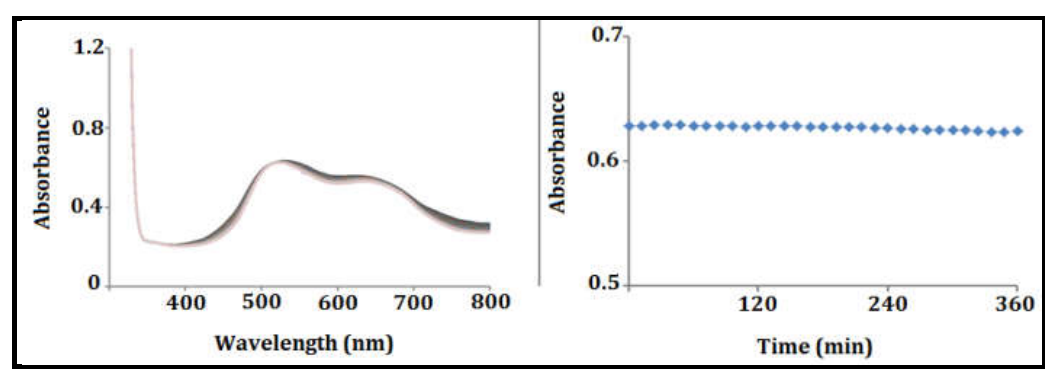

Figure 3. UV-vis analysis of decolourization of the purple solution (W) in acetone at $10 \mathrm{~min}$ time interval $\left(\lambda_{\max }=525 \mathrm{~nm}\right)$.

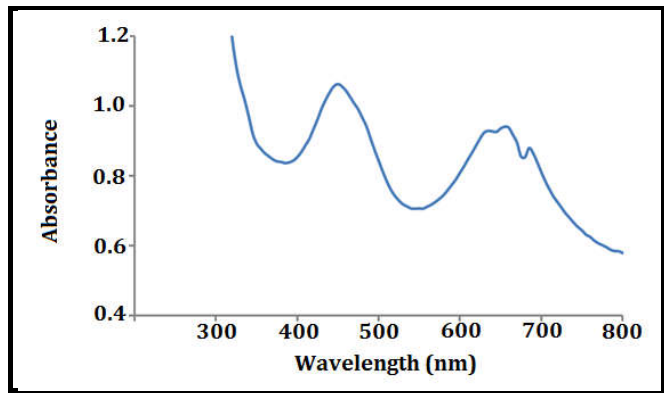

Figure 4. UV-vis analysis of the green chromium metaphosphate solutions in deionised water obtained after fusion with sodium phosphate. 


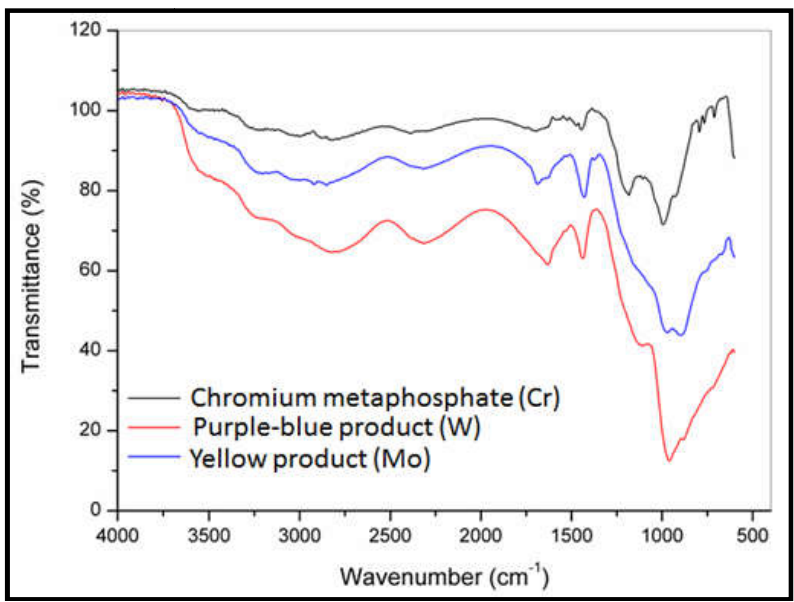

Figure 5. Comparison of IR spectra of the green chromium metaphosphate, yellow molybdenum and purple-blue tungsten products.

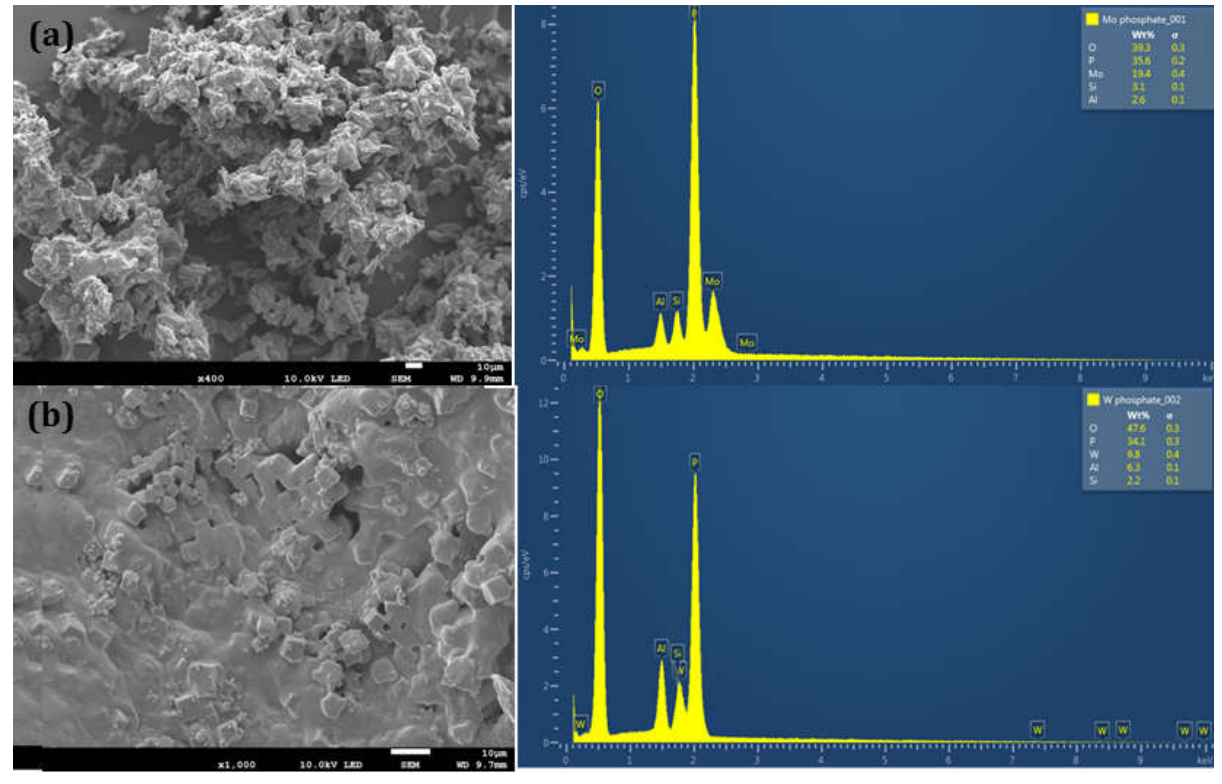

Figure 6. SEM-EDX graphical images of (a) molybdenum and (b) tungsten products. 


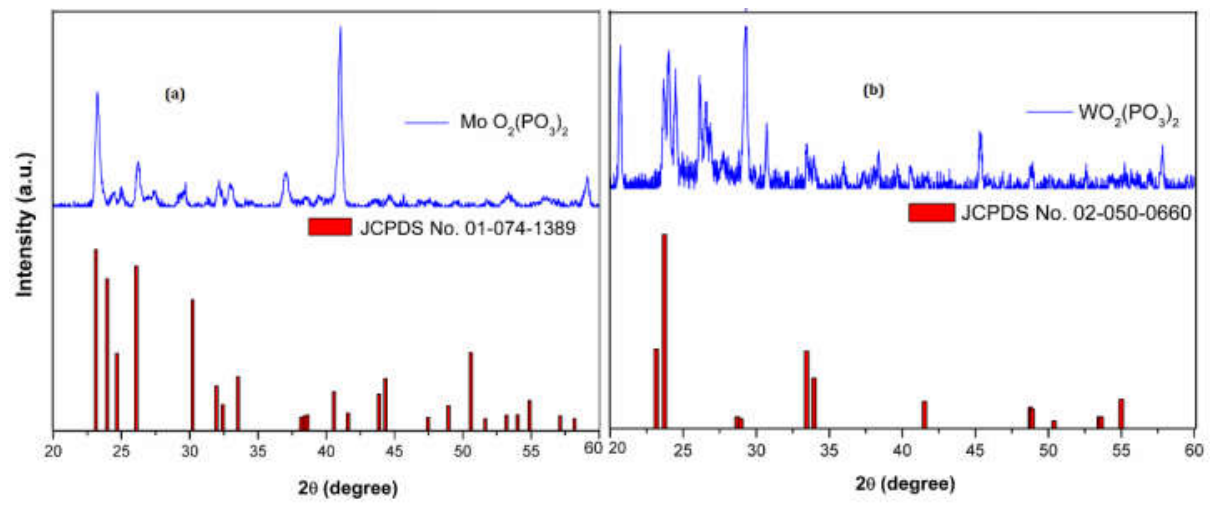

Figure 7. XRD pattern of (a) molybdenum and (b) tungsten products corresponding to the standard pattern of $\mathrm{MoO}_{2}\left(\mathrm{PO}_{3}\right)_{2}$ and $\mathrm{WO}_{2}\left(\mathrm{PO}_{3}\right)_{2}$, respectively.

\section{RESULTS AND DISCUSSION}

Analysis of molybdenum and tungsten products obtained after fusion with ammonium phosphate flux

Fusion of molybdenum powder and tungsten oxide with ammonium phosphate salt as flux (ratio 1: 25 respectively) was done as described by Chiweshe et al. [10]. The view was to determine if ammonium phosphate salt can be used as flux in the synthesis of molybdenum and tungsten metaphosphate compounds. The formation of yellow [14] and purple-blue melts of molybdenum and tungsten respectively were the early indication of a successful conversion of the starting material to the products. The molybdenum and tungsten glassy melts were soluble in deionized water except the chromium melt (Scheme 1). The resultant yellow and purple-blue solutions of molybdenum and tungsten decolorize after standing overnight at room temperature. The decolorization of both solutions was unexpected and pointed to the possible chemical similarities between the two products. These color changes were different from the chromium results, which suggest a possible shift in chemical properties of group VIB elements with ammonium phosphate flux. These preliminary findings prompted further investigations on the stability of molybdenum and tungsten products.

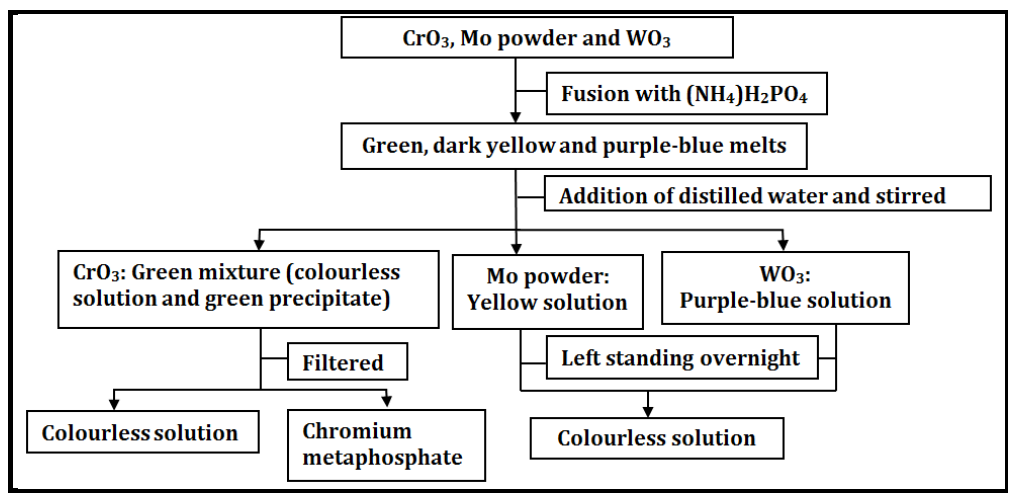

Scheme 1. Comparison of the fusion procedure of chromium, molybdenum and tungsten with ammonium phosphate flux. 
$U V$-vis characterization of molybdenum and tungsten products in deionised water, methanol and acetone

UV-vis analysis of the yellow (Mo) and purple-blue (W) solutions were compared with the UVvis spectrum of the isolated chromium metaphosphate compound to determine any similarities in absorption peaks. Chromium metaphosphate was insoluble in non polar, polar organic solvents and strong acidic/basic medium. Dissolution of the isolated chromium metaphosphate compound was achieved through fusion with excess amount of sodium phosphate flux. The absorbance spectrum of the resultant green solution (Figure 4) showed two distinguished peaks in the region of 455 and $664 \mathrm{~nm}$ that were similar to the spectrum of chromium(III) chloride which confirmed the presence of $\mathrm{Cr}^{3+}$ species [15]. No discoloration of the green chromium solution was observed.

UV-vis analysis of the yellow and purple-blue solutions in deionised water (Figure 1) showed maximum absorbance $\left(\lambda_{\max }\right)$ at 420 and $515 \mathrm{~nm}$ for molybdenum and tungsten respectively. Rapid decrease in absorbances (decolourization) of both solutions occurred within $\sim 3$ hours. These changes in color further suggests the instabilities of both molybdenum and tungsten products. Further analysis of the influence of solvents was performed using methanol (Figure 2) and acetone (Figure 3) which were previously found to dissolve both molybdenum and tungsten melts. UV-vis analysis results of molybdenum solution in methanol revealed gradual decrease in the absorption spectrum ( $\sim 5$ hours decolorization time) compared to tungsten solution in the same solvent ( $\sim 3$ hours). However, decolourazation of tungsten solution was slower in acetone ( $\sim 5$ hours) which affirmed the influence of solvents towards the stability of molybdenum and tungsten products. Deecolorization time between molybdenum and tungsten solutions varied depending on the type of solvent used (Table 1).

Table 1. UV-vis analysis of the influence of solvents in the decolorization of molybdenum and tungsten products.

\begin{tabular}{|c|c|c|c|}
\hline Products & Solvent & Absorbances $\left(\lambda_{\max }\right)(\mathrm{nm})$ & Time taken to decolourize (hours) \\
\hline Chromium & Deionized water & 455 and 664 & - \\
\hline \multirow{2}{*}{ Molybdenum } & Deionized water & 415 & 3 \\
\cline { 2 - 4 } & Methanol & 420 & 5 \\
\hline \multirow{3}{*}{ Tungsten } & Deionized water & 535 & 3 \\
\cline { 2 - 4 } & Methanol & 515 & 3 \\
\cline { 2 - 4 } & Acetone & 525 & 5 \\
\hline
\end{tabular}

No significant changes were observed for both molybdenum and tungsten solutions using dried solvents (methanol and acetone). However, decolourization using methanol and acetone occurred from top to bottom contrary to the uniform decolourization observed using deionised water. This remarkable observation revealed the possibility of air (oxygen) influence in the decolorization of both products. Molybdenum and tungsten solutions kept in a desicator filled with nitrogen showed no decolorization.

Characterization of molybdenum and tungsten products using IR, SEM-EDX and XRD analysis

IR characterization. The IR spectrum of isolated solid molybdenum and tungsten solid products were compared against each other to determine any similarities or differences in the stretching frequencies. Both spectra were compared with the IR spectrum of chromium metaphosphate to determine the presence of any corresponding characteristic metaphosphate peaks in the spectra of molybdenum and tungsten products. All the IR spectra showed similar stretching frequencies in the region of 1250-1690 and 898-979 $\mathrm{cm}^{-1}$ which were assigned to the stretching antisymmetric vibration of $\mathrm{P}-\mathrm{O}$ groups (Table 2). Also present was the small peaks in the region 
in the region of $718-748 \mathrm{~cm}^{-1}$ were ascribed to the symmetric vibration of the P-O groups [16]. The visible broad band bearing two minor peaks in the region of $1118-1130$ and $1040-1043 \mathrm{~cm}^{-1}$ are due to the simultaneous existence of $\mathrm{PO}_{2}$ and $\mathrm{PO}_{3}$ groups in the metaphosphates compounds [6]. The presence of the characteristic peaks in both products was a clear indication of the presence of ametaphosphate compound.

Table 2. Infrared stretching frequencies of molybdenum and tungsten products compared with the stretching frequencies of the isolated chromium metaphosphate.

\begin{tabular}{|c|c|c|c|}
\hline & \multicolumn{3}{|c|}{ Stretching frequencies $\left(\mathrm{V}_{\mathrm{cm}}{ }^{-1}\right)$} \\
\hline Assigned group & Chromium metaphosphate & Molybdenum product & Tungsten product \\
\hline $\mathrm{V}_{\mathrm{as}}(\mathrm{P}-\mathrm{O})$ & 1254 & 1447 & 1690 \\
\hline $\mathrm{V}_{\mathrm{s}}(\mathrm{P}-\mathrm{O}) \mathrm{PO}_{2}$ & 1127 & - & - \\
\hline $\mathrm{V}(\mathrm{P}-\mathrm{O}) \mathrm{PO}_{3}$ & 1040 & 1041 & 1043 \\
\hline $\mathrm{V}_{\mathrm{s}}(\mathrm{P}-\mathrm{O})$ & 962 & 898 & 860 \\
\hline $\mathrm{V}_{\mathrm{s}}(\mathrm{P}-\mathrm{O}) \mathrm{P}-\mathrm{O}-\mathrm{P}$ & 768 & 883 & 895 \\
\hline $\mathrm{V}_{\mathrm{s}}(\mathrm{P}-\mathrm{O})$ & 718 & - & - \\
\hline
\end{tabular}

as - antisymmetric, s - symmetric, - peak not available.

SEM-EDX characterization. Determination of the chemical composition of molybdenum and tungsten products was done using SEM-EDX analysis. Crystalline particles of molybdenum and irregular amorphous-like particles of tungsten product were shown using SEM technique (Figure 6). EDX qualitaitive analysis of molybdenum product showed the presence of Mo, P, O, $\mathrm{Si}$ and $\mathrm{Al}$ whilst tungsten product showed the presence of $\mathrm{W}, \mathrm{P}, \mathrm{O}, \mathrm{Ca}$ and $\mathrm{Na}$. The presence of impurities in these products were determined to be from the ceramic crucible which was chemically attacked during the fusion process.

Determination of the chemical composition of both molybdenum and tungsten products was performed using EDX analysis (Figure 6). The increase in percentage weight of elements in molybdenum and tungsten products were in the order of $\mathrm{M}$ (Mo and $\mathrm{W}$ ) $<\mathrm{P}<\mathrm{O}$. Molybdenum product showed 7.1\%wt (Mo), 37.2\%wt (P) and 53.5\%wt (O) whilst tungsten product showed $10.3 \%$ wt $(\mathrm{W}), 24.7 \% \mathrm{wt}(\mathrm{P})$ and $46.5 \% \mathrm{wt}(\mathrm{O})$. The stoichometric quantities (\%wt) of each element in the product equated to the presence of polyphosphate compound with a chemical formula, $\mathrm{Mo}_{2}\left(\mathrm{PO}_{2}\right)_{5}$ and $\mathrm{W}_{2}\left(\mathrm{P}_{5} \mathrm{O}_{9}\right)$, respectively.

$X R D$ characterization. The phase formation and crystalline nature of the molybdenum and tungsten solid products was determined using XRD patterns (Figure 7). The poorly-defined XRD pattern of molybdenum was consistent with the pattern obtained by Haushalter and Mundi [3] which reveals impure phases of molybdenum polyphosphate products. The XRD pattern of tungsten product was characterized by numerous background noise, which signified an amorphous like product that also was consistent with the SEM images. The room temperature XRD patterns of both molybdenum and tungsten products were matched using the standard XRD patterns in the International Centre for Diffraction Data base or the Joint Committee on Powder Diffraction Standards data base (JCPDS) to determine the corresponding chemical formula. Molybdenum and tungsten patterns matched with the standard $\mathrm{MoO}_{2}\left(\mathrm{PO}_{3}\right)_{2}$ (JCPDS No. 00-050-0660) and $\mathrm{WO}_{2}\left(\mathrm{PO}_{3}\right)_{2}$ (JCPDS No. 01-074-1389) which exhibit orthorhombic and monoclinic structures respectively. The decolourization of the yellow and purple-blue products of molybdenum and tungsten respectively together with the subsequent formation of molybdenum oxide phosphate [17] $\left(\mathrm{MoO}_{2}\left(\mathrm{PO}_{3}\right)_{2}\right)$ and tungsten oxide phosphate [18] $\left(\mathrm{WO}_{2}\left(\mathrm{PO}_{3}\right)_{2}\right)$ was postulated to occur as shown in Eqn. 1-3 although no proposition was found in literature that could support this concept. However, several authors have confirmed the formation of the yellow product of molybdenum metaphosphate $[6,13,14]$. 


$$
\begin{aligned}
& \left(\mathrm{NH}_{4}\right) \mathrm{H}_{2} \mathrm{PO}_{4} \stackrel{\Delta\left(800^{\circ} \mathrm{C}\right)}{\longrightarrow} \mathrm{H}^{+}+\mathrm{PO}_{3}^{-}+\mathrm{NH}_{3}+2 \mathrm{H}_{2} \mathrm{O} \\
& 3 \mathrm{PO}_{3}^{-}+\mathrm{M}^{3+} \longrightarrow \quad \mathrm{M}\left(\mathrm{PO}_{3}\right)_{3} \text { (unstable; } \mathrm{M}=\text { Mo and } \mathrm{W} \text { ) } \\
& (\mathrm{pH}=1.3) \\
& \mathrm{M}\left(\mathrm{PO}_{3}\right)_{3}+\mathrm{O}_{2} \text { (exposed to air) } \longrightarrow \mathrm{MO}_{2}\left(\mathrm{PO}_{3}\right)_{2}+\mathrm{HPO}_{3} \\
& \text { (colorless product) }
\end{aligned}
$$

\section{CONCLUSION}

Fusion of powdered molybdenum and tungsten oxide using ammonium phosphate salt as flux (1:25 respectively) yielded soluble products that were different from the chromium metaphosphate product. UV-vis analysis of molybdenum and tungsten solutions in deionized water, methanol and acetone showed the instability (decolourization) of both solutions at room temperature. Decolourization of these solutions was influenced by the solvent type and exposure to air. Both molybdenum and tungsten solutions were stable (no decolourization) in nitrogen gas. Isolation of the solid molybdenum and tungsten products were achieved by drying the solutions using nitrogen gas. SEM-EDX analysis for both products revealed a micro-crystalline product for molybdenum and a highly amorphous product for tungsten. XRD patterns for both molybdenum and tungsten products matched with the standard XRD patterns of molybdenum oxide phosphate $\left(\mathrm{MoO}_{2}\left(\mathrm{PO}_{3}\right)_{2}\right)$ and $\left(\mathrm{WO}_{2}\left(\mathrm{PO}_{3}\right)_{2}\right)$, respectively.

\section{ACKNOWLEDGEMENTS}

Thanks to the Research Fund of the Free State University for the financial support and the Physics department of the University of the Free State for the XRD analysis.

\section{REFERENCES}

1. Durville, F.; Behrens, E.; Powell, R.; Laser-Induced refractive index gratings in Eu-doped glasses. Phys. Rev. B 1986, 34, 4213-4220.

2. Broer, M.; Bruce, A.; Grodkiewicz, W. Phoinduced refractive-index changes in several $\mathrm{Eu}^{3+}$, $\mathrm{Pr}^{3+}$ and $\mathrm{Er}^{3+}$ doped oxide glasses. Phys. Rev. B 1992, 45, 7077-7083.

3. Haushalter, R.C.; Mundi, L. Reduced molybdenum phosphate: Octahedral-terahedral framework solids with tunnels, cages and micropores. Chem. Mater. 1992, 4, 31-48.

4. Murashova, E.V.; Chudinova, N.N; Ilyukhin A.B. Specific features of the crystal structure of erbium polyphosphate of the structural type C. Crystallogr. Rep. 2007, 52, 230-234.

5. IIiva, D.; Kovacheva D.; Petkov, C; Bogachev G. Vibrational spectra of $\mathrm{R}_{(}\left(\mathrm{PO}_{3}\right)_{3}$ metaphosphates ( $\mathrm{R}=\mathrm{Ga}$, In, Y, Sm, Gd, Dy). J. Raman, Spectrosc. 2001, 32, 893-899.

6. Rojo, J.M.; Mesa, J.L.; Lezama, L.; Rojo, T. Spectroscopic and magnetic properties of three $\left(\mathrm{M}\left(\mathrm{PO}_{3}\right)_{3}(\mathrm{M}=\mathrm{Cr}\right.$ and Mo) metaphosphates. J. Mater. Chem. 1997, 7, 2243-2248.

7. Chudinova, N.N. On crystalline of poly-and metaphosphates of rare earth metals. Izv. Akad. Nauk SSSR, Neorg. Mater. 1979, 15, 931-941.

8. Zhou, W.; He, W.; Zhang, X.; Liu, J.; Du, Y.; Yan, S.; Tian, X.; Sun, X; Han X; Yue Y. Simple and rapid Synthesis of $\mathrm{Fe}\left(\mathrm{PO}_{3}\right)_{3}$ by Microwave Sintering. J. Chem. Eng. Data. 2009, 54, 2073-2076.

9. Kinnaird, J.A.; Kruger, F.J.; Nex, P.A.M.; Cawthorn, R.G. Chromitite formation - a key to understanding processes of platinum enrichment. Appl. Earth Sci. Trans. Institutions Mining Metallurgy: Section B 2002, 111, 23-25. 
10. Chiweshe, T.T.; Purcell, W.; Venter, J.A. Evaluation of PGE liberation and chromium isolation in a solid UG2 chromitite concentrates at moderate temperatures using ICP-OES. $J$. Miner. Metals Mater. Soc. (JOM) 2016, 68, 1691-1700.

11. Gruss, M.; Glaum, R. Refinement of the superstructure of C-type chromium(III) tris(metaphosphate), $\mathrm{Cr}\left(\mathrm{PO}_{3}\right)_{3}$. Acta Cystallogr. Sect. C 1996, 52, 2647-2650.

12. Chiweshe, T.T.; Purcell W. Chromium isolation from different mineral ore. Conference Proceeding of EMC 2017, 1-15.

13. Douglass, R.M.; Staritzky E. Molybdeum metaphosphate $\left(\mathrm{Mo}\left(\mathrm{PO}_{3}\right)_{3}\right)$. Anal. Chem. 1957, 29, 985.

14. Watson, I.M.; Borel, M.M.; Chardon, J.; Leclaire, A. Structure of the trivalent molybdenum metaphosphate $\mathrm{Mo}\left(\mathrm{PO}_{3}\right)_{3}$. J. Solid State Chem. 1994, 111, 253-256.

15. Abbott, A.P.; Al-Barzinjy, A.A.; Abbott, P.D.; Frisch, G.; Harris, R.C.; Hartley, J.; Ryder, K.S. Speciation, physical and electrolytic properties of eutectic mixtures based on $\mathrm{CrCl}_{3} \cdot 6 \mathrm{H}_{2} \mathrm{O}$ and urea, Phys. Chem. Chem. Phys. 2014, 16, 9047-9055.

16. Stuart, B. Infrared Spectroscopy, Fundamentals and Applications, John Wiley and Sons: Sydney, Australia; 2004; p 84.

17. Kierkegaard, P. Molybdenum oxide phosphate. Ark. Kemi 1961, 18, 521-532.

18. Mioc, U.; Dimitrijevic, R.; Davidovic, M.; Nedic, Z.; Mitrovic, M.; Colomban, P.H. Tungsten oxide phosphate. J. Mater. Sci. 1994, 29, 3705-3718. 